\title{
Cerebral vein and sinus thrombosis
}

\author{
Julia Röper-Kelmayr ${ }^{1 *}$, Franz A. Fellner ${ }^{1,2}$, Lukas Kellermair ${ }^{3}$ and Veronika Leeb ${ }^{1 *}$ \\ ${ }^{1}$ Central Radiology Institute, Kepler University Hospital, Medical Faculty of the Johannes Kepler University, Linz, Austria \\ ${ }^{2}$ Medical Faculty of the Friedrich-Alexander-University of Erlangen-Nürnberg, Erlangen, Germany \\ ${ }^{3}$ Department of Neurology II, Kepler University Hospital, Medical Faculty of the Johannes Kepler University, Linz, Austria
}

\section{Case report}

This report discusses the case of a 32-year-old male patient who came into our Department of Neurology complaining of repeated severe headaches with symptom-free intervals of increasingly shorter duration between episodes.

Due to the excruciating pain the patient was in at the time of his arrival, acute imaging via non-enhanced computed tomography of the head (CCT) was performed to preclude hemorrhage (Figure 1).

The resulting images showed a distension and hyperdense appearance of the supra- and infratentorial dural sinuses as well as of cerebral veins, whereupon a cerebral vein and sinus thrombosis was diagnosed.

Upon clinical deterioration of the patient's condition including renewed onset of formication in the left upper extremity, contrastenhanced magnetic resonance (MR) imaging of the cerebrum was performed (Figures 2-4). This provided an even more detailed depiction of the extent of the vein and sinus thrombosis. This examination made it possible to rule out complications such as ischemia, bleeding and cerebral edema.

Typically, patients with cerebral sinus and vein thrombosis are young females. In addition to genetic prothrombotic predisposition, the use of oral contraceptives, nicotine abuse, pregnancy, and metabolic disorders (thyrotoxicosis, dehydration) also increase the risk of developing sinus sinus and vein thrombosis [1-4].

Sinus and vein thromboses can also accompany CNS infections and CNS trauma or can occur paraneoplastically $[2,4]$.

Following initial therapy with low-molecular-weight heparin and observation in the ICU, thrombophilia diagnostics were performed on our patient, which revealed a homozygous PAI-1 4G/5G polymorphism. Thus, the cause of our patient's heightened predisposition to develop

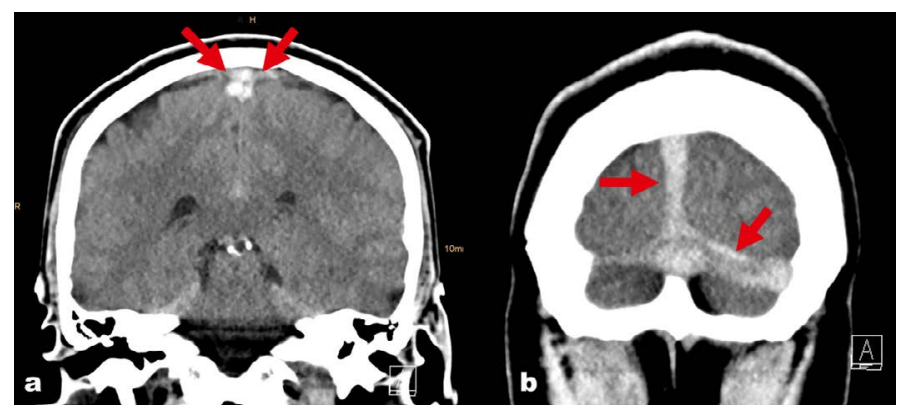

Figure 1 a,b. Hyperdense, dilated superior sagittal sinus and transverse sinus in nonenhanced CCT images.

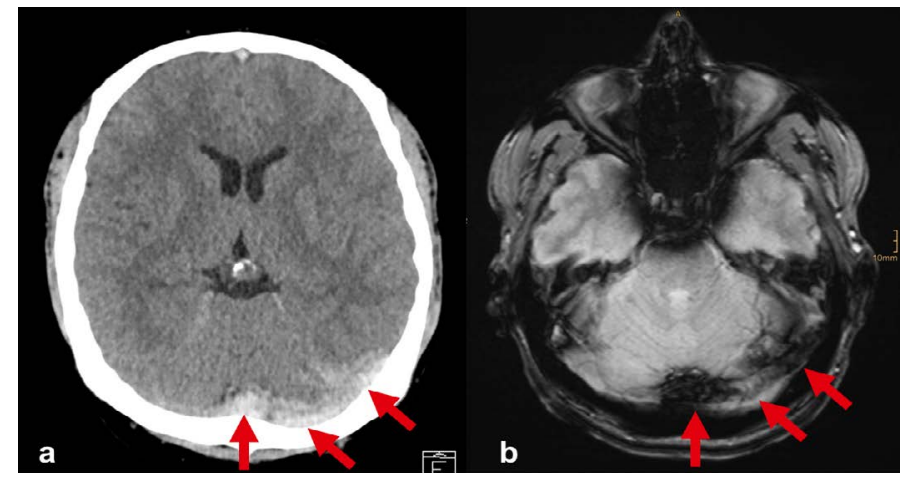

Figure 2. Sinus thrombosis; a) non-contrast-enhanced cranial CT. hyperdense superior sagittal sinus and left transverse sinus. b) hypointense appearance of the thrombus in the $\mathrm{T} 2 *$-weighted gradient-echo sequence from the consecutive MR scan performed on the patient.

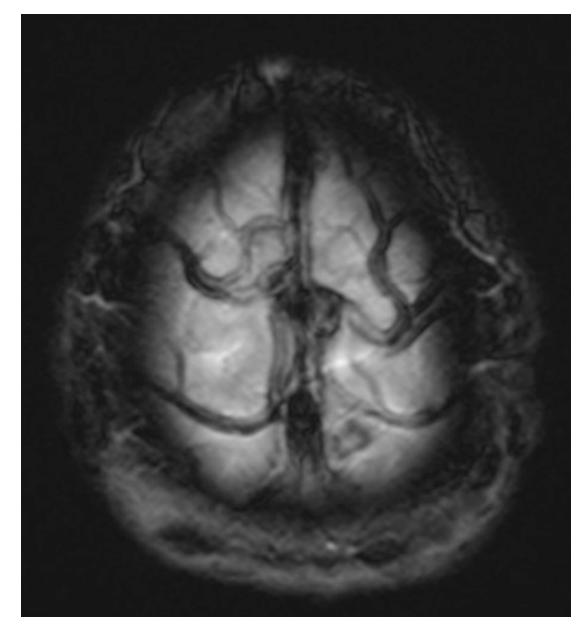

Figure 3. MR examination; the $\mathrm{T} 2 *$-weighted gradient-echo sequence shows multiple thrombosed veins on the surface of the brain (hypointense signal due to the presence of deoxyhemoglobin).

Correspondence to: Julia Röper-Kelmayr, Central Radiology Institute, Kepler University Hospital, Medical Faculty of the Johannes Kepler University, Linz, Austria, E-mail: julia.roeper-kelmayr@kepleruniklinikum.at

Veronika Leeb, Central Radiology Institute, Kepler University Hospital, Medical Faculty of the Johannes Kepler University, Linz, Austria, E-mail: veronika.leeb@ kepleruniklinikum.at

Key words: cerebral venous thrombosis, computed tomography, magnetic resonance imaging

Received: October 20, 2017; Accepted: November 03, 2017; Published: November 06, 2017 


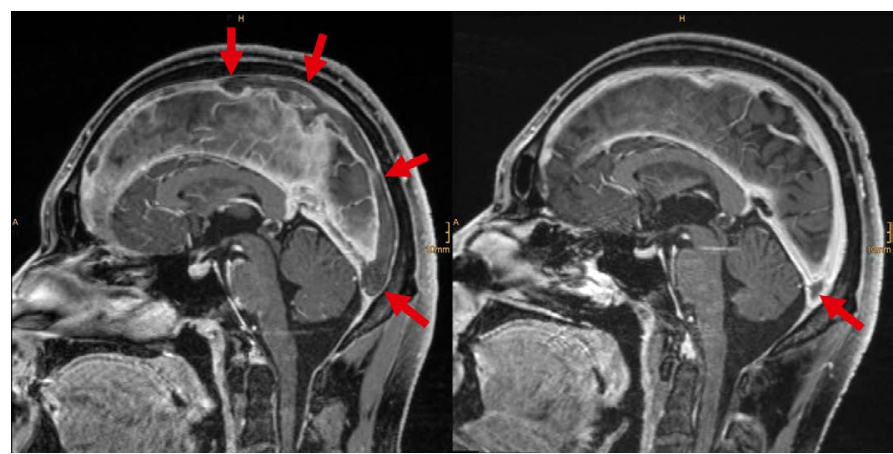

Figure 4. MR examination. contrast-enhanced T1-weighted 3D gradient-echo data set (MP-RAGE) shows a contrast medium filling defect (empty delta sign) in the superior sagittal sinus and Sinus rectus due to dural sinus thrombosis.

thrombosis-in addition to his known abuse of nicotine-was discovered.

After three months, the response to the anticoagulative therapy was confirmed by contrast-enhanced magnetic resonance imaging of the cerebrum, which showed the thrombus formations' original dimensions to have receded considerably.

Since the clinical picture of a sinus and vein thrombosis is often very unspecific and genetic predispositions are not known in many cases, particular emphasis is placed on diagnosis by means of imaging procedures. A delayed diagnosis can be accompanied by complications in the form of infarction, bleeding due to venous congestion, and brain edema leading to death $[2,4]$.
Imaging characteristics of a sinus \& venous thrombosis in unenhanced CT scans of the cerebrum, which are frequently performed as the initial form of examination to determine the cause of headaches, are: hyperdense, dilated sinus (dense triangle sign); congested hyperdense bridging veins (cord sign).

In the performance of differential diagnostics, consideration must be given to acute subdural hematoma and elevated hematocrit levels.

Contrast-enhanced MR imaging can determine the precise age of the thrombus in addition to its exact dimensions. Fresh thromboses appear isointense to the cortex in the T1-weighted and hypointense in the T2-weighted images. The possible complications such as ischemia and cerebral edema are more clearly apparent in images generated by MR than by CT. Hypoplasia or aplasia of the sinus and giant arachnoid granulations can pose differential diagnostic difficulties [4].

\section{Disclosure}

No conflicts of interest. No grants or financial supports.

\section{References}

1. Knauth MG (2011) Headaches - more than sinusitis. Radiologie 3: 247-264.

2. Poon CS, Chang J, Swarnkar A, Johnson M, Wasenko J (2007) Radiologic diagnosis of cerebral venous thrombosis: Pictorial review. AJR Am J Roentgenol 189: 64-75. [Crossref]

3. Huh PW, Cho KS, Yoo DS, Kim JK, Kim DS, Kang JK (2002) Deep cerebral venous thrombosis. Acta Neurochir (Wien) 144: 103-105.

4. Osborn AG (2013) Brain - Imaging, pathology, and anatomy. 223-234.

Copyright: $(2017$ Röper-Kelmayr J. This is an open-access article distributed under the terms of the Creative Commons Attribution License, which permits unrestricted use, distribution, and reproduction in any medium, provided the original author and source are credited. 